\section{Formación pedagógica e investigativa en la Licenciatura de Educación Especial de la Universidad Pedagógica Nacional}

\section{Resumen}

El artículo presenta una reflexión en torno a la importancia de fortalecer la formación pedagógica e investigativa de los licenciados en Educación Especial de la Universidad Pedagógica Nacional. Desde conceptos y aportes del grupo "Historia de la práctica pedagógica", así como desde el análisis de diversos autores contemporáneos se propone una mirada que instaure nuevos saberes y cree una ruptura con estructuras de conocimiento históricamente normalizadas. Esta mirada contempla la realización de trabajos investigativos de corte histórico y documental que de cuenta de la historia de la educación especial como parte de la historia general de la educación y la pedagogía.

Palabras clave: Educación especial, pedagogía, investigación, formación de docentes.
Gloria Janneth Orjuela Sánchez

* Docente de tiempo completo en el Proyecto Curricular de Licenciatura de Educación con énfasis en Educación Especial de la Universidad Pedagógica Nacional. Candidata a Magister en Educación con énfasis en Historia de la Educación y la Pedagogía. Especialista en Gerencia Social de la Educación. Lic. En educación Especial con Énfasis en Retardo en el Desarrollo. Miembro del grupo de investigación: “Filosofía, Sociedad y Educación”, clasificado en categoría D en Colciencias.

Correo electrónico: glojao1@gmail.com.

Fecha de Recepción: 13 de abril de 2009

Fecha de aprobación: 26 de junio de 2009

Pedagogic and research formation at Licenciature in Education with emphasis on Special Education at National Pedagogic University

\section{Abstract}

This article presents a reflection about the importance of strengthening pedagogic and research formation of future graduates in Education with emphasis on Special Education at National Pedagogic University. From concepts and contributions of the group of "History of Pedagogic Practicum", whereas from the analysis of different contemporary authors, it is proposed a look that establishes new knowledge and creates a breaking-off of structures of knowledge normalized historically. This look envisages the carrying out of historical and documentary research works, that tells history of Special Education as a part of General History of Education and Pedagogy.

Key words: Special education, pedagogy, research, formation of teachers.

\section{A formação pedagógica e pesquisa na licenciatura de Educação Especial da Universidad Pedagógica Nacional}

\section{Resumo}

No artigo se reflexiona sobre a importância de robustecer a formação pedagógica dos licenciados e a pesquisa na licenciatura em Educação Especial da Universidad Pedagógica Nacional. Baseados nos conceitos e contribuições do grupo de História da Prática Pedagógica, bem como na análise de autores contemporâneos, os autores propõem uma olhada que introduza novos conhecimentos e acabe com estruturas de conhecimento normalizadas historicamente. Esta visão procura desenvolver pesquisas de tipo histórico e documental sobre historia da Educação Especial como parte da história geral da educação e da pedagogia.

Palavras chave: Educação especial, pedagogia, pesquisa, formação de professores. 
as dinámicas que en educación impone el discurso de la inclusión -han implicado grandes cuestionamientos y cambios, no sólo para la educación especial; también se están generando notables transformaciones en la formación inicial y permanente de los licenciados. De acuerdo con los discursos actuales, se requiere ahora más que nunca una formación que responda a las ideas de una educación y una escuela inclusivas.

En ese sentido, se puede pensar que los pilares fundamentales para la formación de un educador especial deben estar dados por dos ejes: la formación pedagógica, que comprende los discursos y las prácticas, y la formación investigativa. Vale la pena aclarar que tanto los discursos y las prácticas pedagógicas como la formación investigativa están estrechamente relacionados: de una intensa y apropiada formación pedagógica dependerá, en gran medida, la motivación para adentrarse en caminos y alternativas investigativas diversas. Dicha formación le permitirá al educador especial ver/pensar su papel como maestro y ver/pensar la educación especial de otro modo.

En busca de esa transformación para los licenciados de Educación Especial, las prácticas se convierten en un aspecto fundamental, pues cada situación práctica se constituye en un momento de intenso análisis investigativo que utiliza los elementos y las herramientas que el saber pedagógico le ha podido aportar. En ese orden de ideas, Noguera C. (2005) plantea dos formas de acción para analizar la dicotomía existente entre teoría y práctica. Por un lado, se remite al concepto de aplicar, que puede definirse como la sobre posición de una cosa sobre la otra o el esfuerzo y empeño que se pone al realizar una acción:

Si tomamos la teoría como aquello opuesto a la práctica o [...] como consecuencia o incluso como inspiradora de la práctica, tendríamos entonces una relación de aplicación. Desde este punto de vista la teoría sería algo incompleto cuyo complemento necesario sería la práctica. Para que fuese válida, la teoría tendría que pasar por la aplicación en la práctica (Noguera, C., 2005. p. 8).
"Para los licenciados de Educación Especial, las prácticas se convierten en un aspecto fundamental, pues cada situación práctica se constituye en un momento de intenso análisis investigativo que utiliza los elementos y las herramientas que el saber
pedagógico le ha podido aportar"

De otro lado, el concepto utilizar se explica como el hecho de sacar provecho o como el simple uso que se hace de algo:

Tomemos ahora la palabra utilizar y consideremos sus dos acepciones: como aprovechar o sacar provecho de una cosa, y como usar [...] desde la utilización [...] tan solo buscaríamos sacar algún provecho o simplemente usar, es decir, servimos, valernos como lo hacemos con cualquier herramienta o instrumento (Noguera, C., 2005, p. 9).

En última instancia, los diversos discursos de la inclusión posibilitan y se convierten en el pretexto perfecto para pensar de otro modo la educación especial, en general, y la formación de educadores especiales, en particular.

\section{La Formación Pedagógica}

Al hablar de formación pedagógica es preciso concretar el concepto de pedagogía para posteriormente situar la mirada en el fenómeno local, más específicamente en la Universidad Pedagógica Nacional. Desde distintos resultados de investigaciones publicadas autores como Zuluga O., Martínez B., Echeverri A., Restrepo S. y Quiceno H., entre otros, coinciden teóricamente en que es necesario reconocer de la pedagogía

[...] su estatuto de disciplina (saber) que [...] posee conceptos, campos de aplicación, problemas propios o planteados a ella por otros saberes, y relaciones con otras disciplinas [...] Que la pedagogía está conformada por un conjunto de nociones y prácticas que hablan del conocimiento, del hombre, del lenguaje de la enseñanza, de la escuela y del maestro [...] Que la pedagogía es la disciplina que conceptualiza, aplica y experimenta los conocimientos referentes a la enseñanza de los saberes... (Zuluaga et. al. 2003, p. 36).

Por ello, la pedagogía se asume como una disciplina en construcción permanente que habla del saber pedagógico, que tiene su propio lugar y que puede armar su propio cuerpo epistemológico. En ese sentido, los elementos constitutivos de la pedagogía se transforman en distintos campos de interés para los licenciados de formación general y, por supuesto, se hacen pertinentes para licenciados en educación especial. En fin, elementos constituidos en factor clave que, desde lo pedagógico, le darán su identidad como sujeto del saber pedagógico en cuanto se enfrente a las prácticas.

Estos campos de interés se relacionan con los discursos - de la didáctica, el currículo, la enseñanza, la educación, etc.--, las instituciones - espacios específicos y delimitados como la escuela, la academia y los lugares privilegiados históricamente como los poseedores del saber pedagógico- y las prácticas -los espacios o ámbitos de la pedagogía
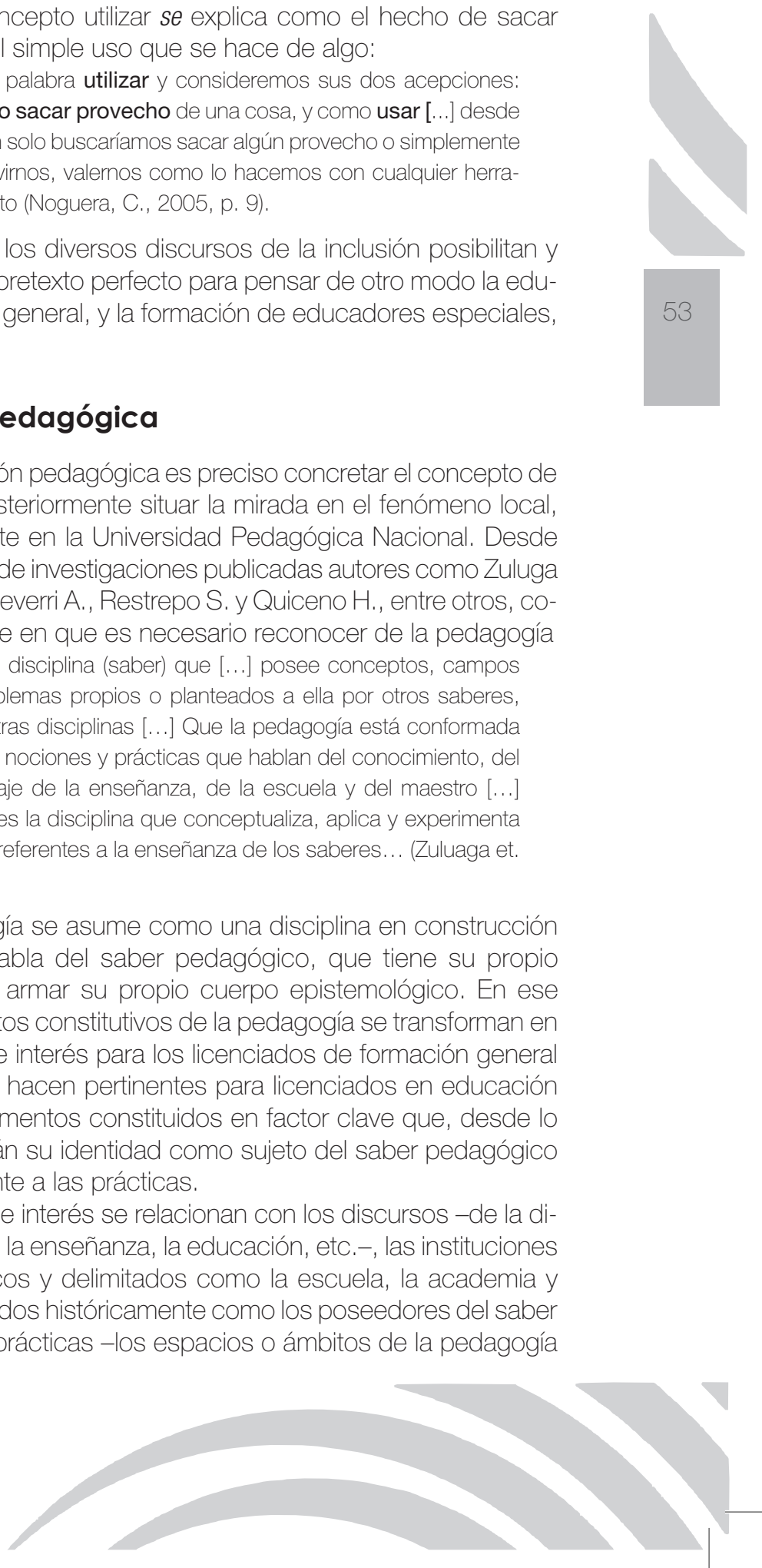
y su campo de aplicación: el discurso-. Lugares y momentos en los que el maestro enfrenta sus conocimientos pedagógicos al discurso de las teorías a través de su instrumento: el método de enseñanza- (Cfr. Zuluaga, 1999).

Dichos campos de interés no se caracterizan por ser estáticos; se encuentran en permanente deconstrucción, construcción, reconstrucción y transformación. Son saberes no doctrinales que emergen de las discusiones y reflexiones realizadas entre los sujetos participantes del saber pedagógico.

Parte de la crisis que vive en la actualidad la disciplina de la Educación Especial se debe a la falta de un piso pedagógico, conceptual y epistemológico consolidado, lo cual la deja a merced y al vaivén de los discursos que van emergiendo: integración, inclusión, diversidad, vulnerabilidad; en fin, saberes que va apropiando y asumiendo de manera apresurada y sin mayores cuestionamientos o sin profundizar en su comprensión.

Así, ello se traduce en los programas de formación de Licenciados en Educación Especial; programas donde la pedagogía se asume con una mirada completamente instrumental y el maestro es visto como aplicador de técnicas de evaluación y/o medición, como adaptador de material y ambientes. De igual manera la pedagogía se asume de manera muy superficial, desde perspectivas, muchas veces naturalizadas, que la llevan a ser abordada de manera emblemática, apegada a los discursos habituales -no se les cuestiona ni se les interroga-.

Al respecto Yarza (2008) citando las ideas de Gallego y Rodríguez, sostiene que éstos programas:

[... s sitúan el papel del maestro en las transformaciones que la sociedad genera en la educación y en la institución escolar desde unas consideraciones prospectivas, es decir, lo que se espera que el maestro sea en el futuro: eficiente, democrático, comunicador, especialista en el aprendizaje, etc. Partiendo de lo anterior, expresan unas indicaciones alrededor de algunas de las más importantes funciones asignadas al profesorado: planificador de la enseñanza, mediador del aprendizaje, gestor del aula, evaluador, investigador e innovador (Yarza, A. 2008, p. 3).

Pero esta crisis en la formación pedagógica del educador especial no sólo es un fenómeno local; también toca los ámbitos internacionales. Cuenta de ello dan algunos documentos de autores diversos como Gallego y Rodríguez (2007), Michels (2005), Blanton, Sindelar y Correa (2006), Peterson (2006) y Arrazola (1996) quienes analizan el asunto desde distintas latitudes del mundo. En general, tales autores coinciden en lo siguiente:

- La filosofía y las ideas del discurso de la inclusión han generado nuevas exigencias formativas tanto a los educadores especiales como a los docentes de aula - o generalistas como los llaman otros autores-.

- La necesidad de un cambio cultural acerca de la forma de mirar y de asumir la diferencia.

- La urgencia de un cambio en la mirada y en el proceso del binomio enseñanzaaprendizaje.

- Un nuevo rol del maestro que se acomode a la coyuntura educativa y que, necesariamente, conduzca a un cambio en las tareas que debe asumir.

- La revisión y el análisis urgente de la formación inicial del profesorado en general.
"Parte de la crisis que vive en la actualidad la disciplina de la Educación Especial se debe a la falta de un piso pedagógico, conceptual y epistemológico consolidado, lo cual la deja a merced y al vaivén de los discursos que van emergiendo: integración, inclusión, diversidad, vulnerabilidad"

- La formación del profesorado vista como la variable de mayor incidencia en la calidad y en el logro de la inclusión educativa.

- El cuestionar la necesidad del énfasis en una formación categorial o genérica, o entre una formación generalista o una específica.

- La organización curricular de los programas de formación de docentes es la expresión de una determinada concepción acerca de la educación especial, de la deficiencia y de las prácticas educativas.

- La formación de educadores especiales ha estado fuertemente permeada por modelos médicos, biológicos, evaluativos y psicológicos que obligan a concentrar la mirada del educador en aspectos de diagnóstico; experiencias que quitan la posibilidad de comprensión de la deficiencia y de la acción pedagógica como un hecho social.

El planteamiento de la crisis mundial que se está viviendo en los programas de formación de educadores especiales y el cuestionamiento que, desde allí, y a través del discurso de la inclusión, se le está trazando a la formación de licenciados debe motivar no solo la reflexión sino la acción. En consecuencia, es esta la oportunidad para proponer maneras distintas de asumir la pedagogía y el saber pedagógico dentro de estos programas de formación.

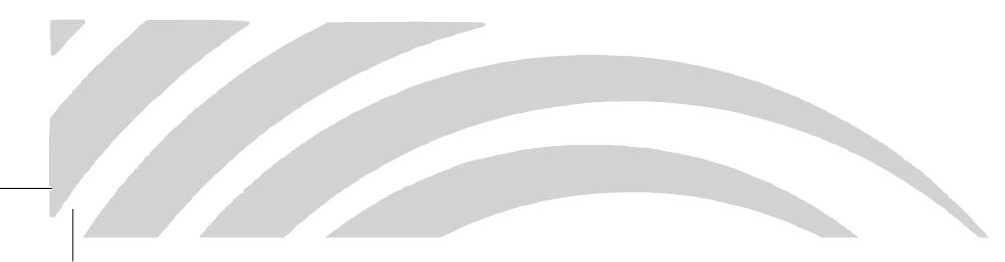




\section{Formación Investigativa}

La formación investigativa, como segundo eje de los pilares de la formación de educadores especiales, se constituye en un elemento clave para poder avanzar y concretar los puntos a favor o en contra del discurso de la educación inclusiva y su aplicación práctica en las aulas regulares. Su valor repercute en las formas de analizar y proponer cambios en los programas de formación de educadores especiales y de docentes en general.

El campo de investigación ha tenido manejos particulares al interior de los programas de formación de docentes; por ejemplo, es trabajado como espacio formativo de manera ambigua y tradicionalista o con tendencia hacia un solo modelo investigativo. Los mismos modelos médicos, biológicos y psicológicos que han imperado en el modo de concebir al anormal, han terminado por influir las investigaciones realizadas en la disciplina de la Educación Especial. El resultado de ello se evidencia en los temas abordados por estudiantes de últimos años en sus trabajos de grado.

En el ámbito mundial se cuenta con reportes de Europa, España y Estados Unidos (Chard 2004, Hegarty 2008 y Cuevas y Saenz 1993) en los cuales se reconoce el valor y la importancia que el área investigativa y científica ha tenido en el campo de conocimiento de la Educación Especial, en específico porque le posibilitó, en sus comienzos, confirmar o rechazar formas de identificación y de enseñanza para estudiantes con dificultades. Sin embargo, los autores también son conscientes del uso limitado que se ha dado a la formación investigativa en las licenciaturas de educación y del uso también restringido y pobre que se ha venido especificando en procesos de investigación. Uso que no ha permitido que dichos estudios tengan el impacto deseado desde lo social.

Asimismo, los autores citados hacen énfasis y resaltan el valor y la importancia del incremento y de la movilización que ha tenido la investigación en las últimas décadas en torno a los aportes del desempeño y la formación de profesionales de la disciplina de la Educación Especial.
De modo puntual, se concentran en la última mitad del siglo pasado, período caracterizado por cambios importantes en conceptos fundamentales de la educación especial, más que en la general. Cambios que han ido de la mano y han contribuido al crecimiento en el estudio académico serio y sistemático de la disciplina en cuestión; estudios que han propiciado y permitido una reorientación de las investigaciones con un desplazamiento de su foco de atención en procesos cognitivos, médicos y psicológicos hacia cuestiones más educativas y sociales. Este cambio de perspectiva ha ampliado el abanico de posibilidades y temáticas de investigación: así se ha convertido un desafío para la formación de educadores especiales.

Sin embargo, los autores también reconocen que, aun cuando ahora la gama de temáticas de investigación es más amplia, gran cantidad de estos estudios se siguen concentrando en casos particulares o se constituyen en trabajos de menor escala o no cuentan con la suficiente subvención económica para sus desarrollos. Tal situación no permite que contribuyan de manera significativa en el fortalecimiento del cuerpo epistemológico y del saber de la educación especial.

De igual modo, para los autores es claro, a partir de las coincidencias en la percepción de la investigación en educación especial, que la finalidad de estos trabajos debe ser la ayuda en la toma de decisiones institucionales respecto de los procesos de inclusión educativa y del aporte que puedan dar a la motivación y a la comprensión necesarias para intervenciones más adecuadas.

Desde la perspectiva nacional, existe un trabajo de análisis y propuesta bastante interesante para la formación de educadores especiales en el país. El profesor Alexander Yarza (2008), desde su experiencia como docente formador en la Universidad de Antioquía, plantea la importancia de hacer apertura hacia escenarios diferentes para la formación del profesorado. En particular, para el área investigativa propende por el abordaje de lo que él denomina unos "análisis de contradicciones" presentes en las prácticas pedagógicas y asociadas a conceptos tradicionales y a los nuevos discursos emergentes; identifica "polos opuestos" o "binomios constitutivos" que permitirían transformar las experiencias y las prácticas.

Para llevar a cabo dicha transformación en la formación del educador especial, el autor plantea algunos elementos básicos:

En un primer movimiento, pretende vernos como un objeto de análisis, es decir, como un agente históricamente condicionado por la historia de la cultura, la educación y la pedagogía (en un tiempo y una geografía determinada). En un segundo movimiento, la reflexividad indica no hacer un análisis exclusivo sobre las formas de hacer o "lo que se hace" (tal como impera en las prácticas formativas de docentes), sino que además sugiere efectuar un análisis de sí mismo: mis representaciones, imaginarios, condiciones sociales, adscripciones conceptuales, creencias, estereotipos, prejuicios, etc. Además, la analítica de las prácticas podría mostrar los objetos de saber y las conceptualizaciones que producen las prácticas y son producidas por las prácticas mismas. La reflexividad no se sitúa exclusivamente en el espacio de las formas de hacer, sino también en las subjetividades y en las conceptualizaciones (Yarza, A. 2008, p. 7).

Dicha postura posibilita, entre otras cosas, que el educador especial se comprenda como sujeto portador y productor de saber pedagógico-didáctico y disciplinar; que los planes de formación establezcan vínculos y relaciones reales y eficaces entre las prácticas docentes 
pertinencia de la formación impartida y de las proyecciones de la misma en el marco del discurso de una educación inclusiva.

En ese orden de ideas, las discusiones y el trabajo del colectivo de maestros del proyecto curricular han confluido en varias aplicaciones prácticas que han empezado a funcionar en los tres primeros semestres; vale la pena aclarar que la efectividad de las mismas dependerá, en gran medida, de que las decisiones sean resultado de consensos y de un trabajo en equipo y mancomunado del total de los profesores. Se hace válido entonces destacar algunas de las concreciones prácticas y, además, proponer algunos aspectos que aún se pueden incluir para complementar los adelantos alcanzados hasta la actualidad:

Núcleo común de pedagogía. Se constituye en una formación generalista para todos los docentes en formación del Departamento. Su campo de interés es la pedagogía, vista como elemento constitutivo e identificador de la esencia en la formación del profesorado. En ese sentido, se propone dar inicio al funcionamiento del núcleo a partir de los espacios: "Historia de la educación y la pedagogía" y "Corrientes pedagógicas". A la anterior propuesta se podrían anexar otros espacios como "Epistemología de la pedagogía y educación y cultura".

Renovación curricular en la Licenciatura en Educación Especial. Para la formación específica del profesor, se concluyó en la posibilidad de dos espacios de evolución y tendencias en la educación especial. Tal opción permite estudiar de manera detallada la historia y la actualidad de la disciplina de la educación especial. A esta propuesta se podría añadir un nuevo espacio que analice la epistemología en educación especial.

Investigación. Reestructurar y movilizar las líneas de investigación del proyecto curricular mediante apertura de nuevas opciones y campos de indagación en el ámbito de la educación
"Dentro de la formación pedagógica se hace inminente, por un lado, el rescate de una epistemología de la Educación Especial que piense cuestiones como la otredad, la mismidad, la normalidad, la anormalidad, la completud, la mirada o pregunta hacia el sujeto, entre otros"

especial. Además, han de vincularse de manera más directa y efectiva los procesos de práctica pedagógica y la elaboración de proyectos de grado de los estudiantes. Sin olvidar una fuerte formación en distintas alternativas o métodos investigativos en los cuales los estudiantes tengan la opción de escoger el que más convenga al contexto en el cual se desarrollará su indagación. Asimismo, en este espacio es pertinente abrir la posibilidad de realizar otro tipo de investigaciones que no necesariamente se encuentren vinculados a las instituciones y prácticas instrumentales -como ha sido manejado de manera tradicional- y que permitan optar por otros tipos de trabajos de corte más analítico, histórico y documental. Un ejemplo de ello sería una línea de investigación acerca de la historia de la educación especial, como elemento constitutivo e importante de la gran historia de la educación y la pedagogía del país.

Para concluir, se considera importante insistir en la fortaleza de la formación pedagógica e investigativa de los educadores especiales como elementos fundamentales que dan la identidad al maestro como sujeto constructor de saber pedagógico y como sujeto constituido históricamente.

En particular, dentro de la formación pedagógica se hace inminente, por un lado, el rescate de una epistemología de la Educación Especial que piense cuestiones como la otredad, la mismidad, la normalidad, la anormalidad, la completud, la mirada o pregunta hacia el sujeto, entre otros. Aspectos que, en su conjunto, fortalecerían de manera muy positiva el piso de saber de la disciplina.

Por otro lado, ha de reconocerse la importancia que se le brinde al abordaje de la historia de la educación especial, pero no una historia lineal y del monumento, sino una historia que logre dar cuenta de los procesos de emergencia de la educación Especial vinculados a la constitución general de los sistemas de educación pública y, por ende, a la historia general de la educación y la pedagogía. Una historia que interrogue acerca de elementos importantes que han sido ejes transversales del devenir de la disciplina: oralidad/lengua de señas; exclusión/ integración/inclusión; feminización del campo, etc. 


\section{Bibliografía}

Blanton, L. P.; Sindelar P. T \& Correa V. I. (2004). Models and measures of beginning teacher quality, en The Journal of Special Education. Vol. 40, Núm. 2, 115-127.

CHARD, D. J. (2004). Maintaining the relationship between science and special education, en Journal of Learning Disabilities. Vol. 37, No. 3, 213-217.

Gallego O., J. L. y Rodríguez, F. A. (2007). «Tendencias en la formación inicial del profesorado en educación especia», en REICE, Revista Electrónica Iberoamericana sobre Calidad, Eficacia y Cambio en Educación, Vol. 5, Núm. 3. [en línea], disponible en: http://www. rinace.net/arts/vol5num3/art12.htm

Gutiérrez Cuevas, P. y Sáenz-Rico De Santiago, B. (1993). «Perspectivas de investigación en educación especial» en Revista Complutense de Educación, 4. 1-8.

HeGarTy, S. (2008), Special education research in europe, en REICE, Revista Electrónica Iberoamericana sobre Calidad, Eficacia y Cambio en Educación Vol. 6, No. 2. [En línea], disponible en: http://www.rinace. net/arts/vol6num2/art13.htm

Vigo Arrazola, M. B. (1997). "Hacia la formación del profesor generalista en educación especial» en Revista Electrónica Interuniversitaria de Formación del Profesorado. España.
Michels, M. H. (2005). "Paradoxos da formação de professores para a educação especial: o currículo como expressão da reiteração do modelo médico-psicológico» en Revista Brasileira Educação Especial, Vol.11, Núm. 2, 255-272.

Noguera, c. (2005). «Prólogo» en Foucault, la pedagogía y la educación. Pensar de otro modo. Bogotá: Magisterio-Universidad Pedagógica Nacional-Instituto para la Investigación Educativa y el Desarrollo Pedagógico (IDEP)-Grupo de la Historia de la Práctica Pedagógica.

Peterson, P. J. (2006). "Inclusão nos Estados Unidos: filosofia, implementação e capacitação de professores» en Revista Brasileira de Educação Especial. Marília, Jan.-Abr. 2006, Vol.12, Núm. 1, 3-10 [en línea], disponible en: http://www.scielo. br/cgi-bin/wxis.exe/iah/

SAlvador MATA, F. (1997). "La educación especial en una escuela Inclusiva» en Revista Profesorado. Vol. 1, Núm. 2, Universidad de Granada.

Universidad Pedagógica Nacional (2006). Acuerdo 035 de 18 de Agosto de 2006

YARZA De Los Rios, A. (2008). «Formación del profesorado en educación especial, inclusión educativa y dispositivos de formación docente» en Revista Colombiana de Educación, 54 [En prensa].

YarzA De Los Rios, A. (2007). Educación y pedagogía de la infancia anormal 1870-1940. Bogotá: Magisterio.

Zuluaga, O. (1999). Pedagogía e historia: la historicidad de la pedagogía. La enseñanza, un objeto de saber. Medellín: Universidad de Antioquia-Anthropos-Siglo del Hombre Editores.

Zuluaga, O. y Otros (2003). Pedagogía y epistemología. Bogotá: Magisterio.

Zuluaga, O. y Otros (2005). Foucault, la pedagogía y la educación: pensar de otro modo. Bogotá: Magisterio-Universidad Pedagógica Nacional-Instituto para la Investigación Educativa y el Desarrollo Pedagógico ( IDEP)-Grupo de la Historia de la Práctica Pedagógica. 


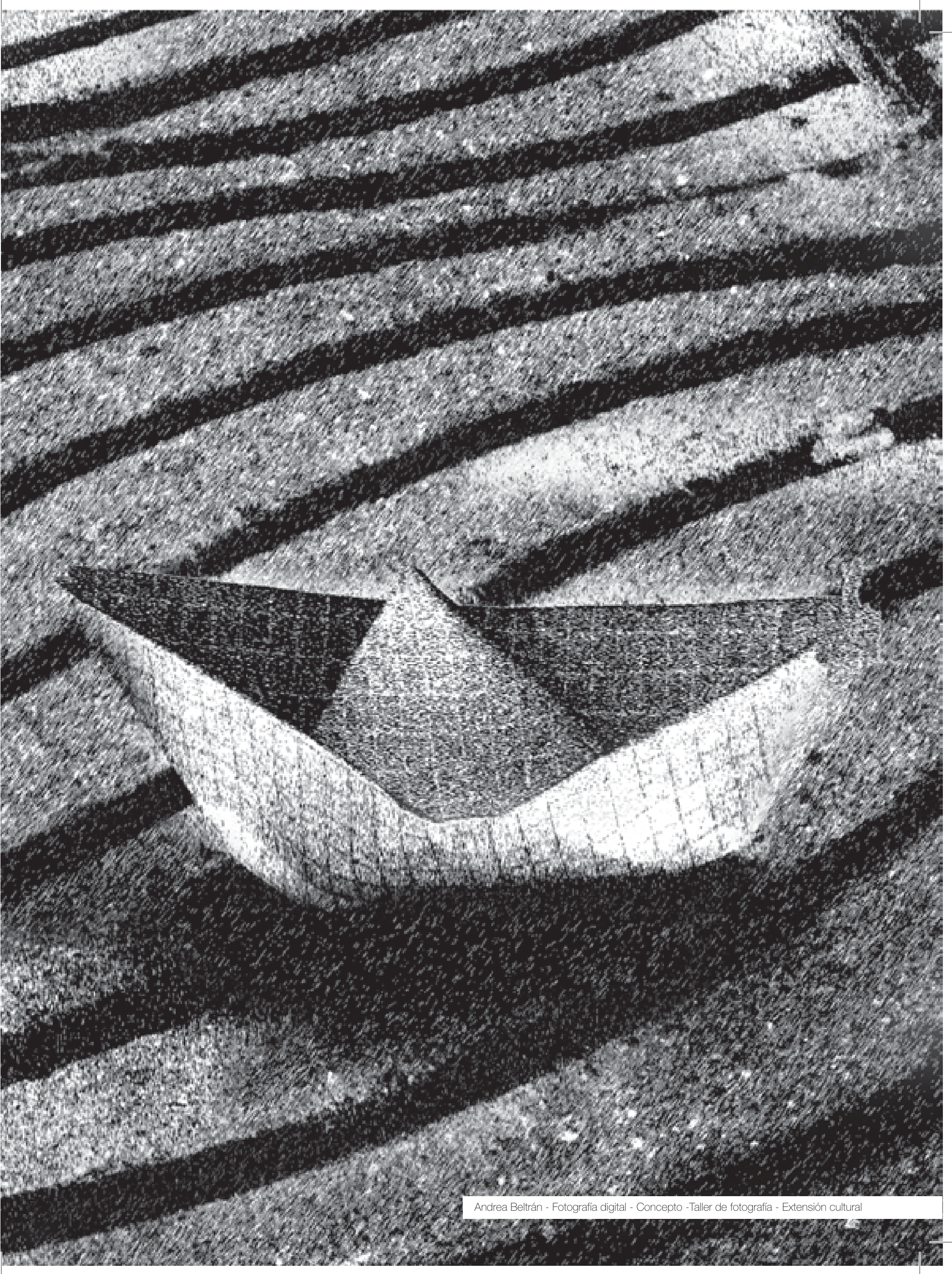

